\title{
Fabrication of Black In203 with Dense Oxygen Vacancy through Dual Functional Carbon Doping for Enhancing Photothermal CO2 Hydrogenation
}

Yuhang Qi

Tianjin University

Jiawei Jiang

Tianjin University

Xichen Liang

University of California

Shuxin Ouyang ( $\square$ oysx@mail.ccnu.edu.cn )

Central China Normal University https://orcid.org/0000-0002-7650-1245

Wenbo Mi

Tianjin University https://orcid.org/0000-0002-9108-9930

Shangbo Ning

Tianjin University

Lei Zhao

University of California

Jinhua Ye

National Institute for Materials Science https://orcid.org/0000-0002-8105-8903

Article

Keywords: indium oxide, carbon doping, oxygen vacancy, photothermal catalysis, $\mathrm{CO} 2$ reduction

Posted Date: November 9th, 2020

DOI: https://doi.org/10.21203/rs.3.rs-102590/v1

License: (c) (i) This work is licensed under a Creative Commons Attribution 4.0 International License. Read Full License 


\section{Abstract}

Photothermocatalytic $\mathrm{CO}_{2}$ reduction as the channel of the energy and environmental issues resolution has captured persistent attention in recent years. $\ln _{2} \mathrm{O}_{3}$ has been prompted to be a potential photothermal catalyst in this sector on account of unique physicochemical properties. However, different from the metal-based photothermal catalyst with the nature of efficient light-tothermal conversion and $\mathrm{H}_{2}$ dissociation, the wide-bandgap semiconductor needs to be modified to possess wide-wavelengthrange absorption and the active surface. It remains a challenge to achieve the two aims simultaneously via single material modulation approach. In this study, one strategy of carbon doping can empower $\ln _{2} \mathrm{O}_{3}$ with two advantageous modifications. The carbon doping can reduce the formation energy of oxygen vacancy, which induces the generation of oxygen-vacancy-riched material. The introduction of oxygen defect levels and carbon doping levels in band gap of $\ln _{2} \mathrm{O}_{3}$ significantly reduces this band gap, which endows it full-spectral and intensive solar light absorption. Therefore, the carbon doped $\ln _{2} \mathrm{O}_{3}$ achieves effective lightto-thermal conversion and delivers a $123.6 \mathrm{mmol} \mathrm{g}^{-1} \mathrm{~h}^{-1}$ of $\mathrm{CO}$ generation rate with near-unity selectivity, as well as prominent stability in photothermocatalytic $\mathrm{CO}_{2}$ reduction.

\section{Introduction}

Energy shortage could be alleviated by fuel regeneration rely on advanced technology rather than exploitation of existing sources

${ }^{1-3}$. Compared with the amount of emitted solar energy in one day, fossil fuel outputs seem negligible $e^{4,5}$. Mitigating the unbalanced supply and demand, photothermocatalytic technology has been proposed as a reasonable attempt ${ }^{6-9}$. On the other hand, $\mathrm{CO}_{2}$ as a well-known excess substance trapped in the atmosphere, could be converted into the value-added product in both greenhouse gas reduction and synthetic chemical generation ${ }^{10-12}$. Typically, high purity $\mathrm{CO}$ from $\mathrm{CO}_{2}$ hydrogenation via reverse water gas shift (RWGS) reaction could be used as raw material for the Fischer-Tropsch synthesis ${ }^{13}$, producing carbonyl-containing compounds ${ }^{14}$, acetamides ${ }^{15}$, triflate electrophiles ${ }^{16}$, and purifying nickel in Mond process ${ }^{17}$. In this way, photothermal catalytic $\mathrm{CO}_{2}$ reduction technology can significantly reduce energy consumption and seems promising for carbon recycle, but the fabrication of high-quality catalyst is not yet mature.

Single-phase $\ln _{2} \mathrm{O}_{3}$ is an impactful thermochemistry catalyst with high selectivity of CO, but its wide-bandgap feature (3.2 eV) leads to unfavorable traits to light absorption (it appears pale-yellow color) and photothermal transformation ${ }^{18,19}$, which limits its application for photothermal catalysis. To extend the limited optical adsorption, researchers make efforts on pretreated $\ln _{2} \mathrm{O}_{3}{ }^{20}$, such as precious metals loading ${ }^{21}$ and nanostructure coating ${ }^{22}$. But the catalytic activity of photothermal $\mathrm{CO}_{2}$ reduction remains low. On the other hand, oxygen vacancy as the active site in $\mathrm{In}_{2} \mathrm{O}_{3}$ plays important role in $\mathrm{CO}_{2}$ reduction reaction, it can powerfully adsorb the $\mathrm{CO}_{2}$ molecules. The unsaturated indium atoms around the oxygen vacancy can gradually dissociate hydrogen inputs to accelerate intermediates formation, then to the products ${ }^{23,24}$. Therefore, to obtain a higher concentration of oxygen vacancy per unit $\ln _{2} \mathrm{O}_{3}$, nanostructures with high specific surface area seem a promising approach ${ }^{25-27}$. Recently, we reported a novel strategy to fabricate ultrathin 2D black $\ln _{2} \mathrm{O}_{3-x}$ nanosheet with full spectrum absorption by photo-induced defect engineering. It exhibits a maximum yield rate of $103.2 \mathrm{mmol} \mathrm{g}_{\mathrm{cat}}{ }^{-1} \mathrm{~h}^{-1}$ and near-unity selectivity for $\mathrm{CO}$ generation ${ }^{28}$. While the oxygen vacancy-rich materials still face the key issue of deactivation due to the refilling of oxygen vacancy during the catalysis process ${ }^{29,30}$. Therefore, how to prepare catalysts of ordinary nano- and micro- particles with comprehensive advantages including superior photothermal conversion efficiency, high concentration of active sites, and long-term stability is still in exploration.

In this work, we report that a dual functional doping of carbon in $\ln _{2} \mathrm{O}_{3}$ through cost-effective hydrothermal synthesis for highly effective photothermal $\mathrm{CO}_{2}$ reduction (Scheme 1a). Experimental data and simulation calculations have consistently confirmed that doped carbon works as an interstitial atom inserted in the lattice, which results in both the bandgap reduction to extend light absorption to the full spectrum and the formation of high-centration and stable oxygen vacancies (Scheme 1b). The widewavelength and intensive light absorption facilitate the effective photothermal conversion $\left(400{ }^{\circ} \mathrm{C}\right.$ of temperature rising in 10 mins under irradiation of $300 \mathrm{~W}$ Xe lamp). Besides, the decrease of formation energy of oxygen vacancy $\left(\mathrm{V}_{\mathrm{o}}\right)$ due to the carbon 
doping enhances the concentration of active sites. The optimized C- $\ln _{2} \mathrm{O}_{3-\mathrm{x}}$ catalyst delivers a CO yield rate of $123.6 \mathrm{mmol}_{\mathrm{cat}}^{-1} \mathrm{~h}^{-}$

${ }^{1}$ (Table S1) and the remarkable stability with no attenuation even after 20 cycles (one hour per cycle). Therefore, manipulation of doped carbon atoms becomes an internal adjustment strategy in inducing oxygen vacancies and then modulating electron structure and surface property in the $\ln _{2} \mathrm{O}_{3}$ system so as to be suitable for photothermal $\mathrm{CO}_{2}$ reduction.

\section{Results}

\section{Fabrication and characterization of carbon doped $\ln _{2} \mathrm{O}_{3}$}

In this experiment, glucose as the carbon source was introduced to prepare carbon doping $\ln (\mathrm{OH})_{3}\left(\mathrm{C}-\ln (\mathrm{OH})_{3}\right)$ nanoparticle precursors (Figure S1 and S2) by hydrothermal reactions. After calcinating in the Ar atmosphere, the $\mathrm{C}-\ln (\mathrm{OH})_{3}$ was dehydrated to form $\mathrm{C}-\mathrm{In}_{2} \mathrm{O}_{3-\mathrm{x}}$, and the morphology remained almost unchanged. Particle size ranged from 50 to $200 \mathrm{~nm}$ and the particle surface was covered many small protuberances (Figure 1a) with uniformly distributed carbon (Figure S3). The $0.177 \mathrm{~nm}$ spacing on the high-resolution transmission electron microscope (HR-TEM) image confirmed the (440) lattice plane in the cubic $\ln _{2} \mathrm{O}_{3}$ crystalline phase corresponding to Fast Fourier transform (FFT) patterns with diffraction along the [110] axis (Figure 1b). The crystal structure was characterized by powder X-ray diffraction (XRD); all the peaks can be indexed into the standard $\ln _{2} \mathrm{O}_{3} \mathrm{PDF}$ card (JCPDS card No.71-2194) (Figure 1c).

In order to investigate the existential form of doped carbon and the relevant structural adjustment in $\ln _{2} \mathrm{O}_{3}$, we simulated the most likely four modalities, including carbon atom substitution for oxygen atom $\left(\mathrm{C}_{\mathrm{s}}-\mathrm{O}\right)$, carbon atom substitution for indium atom at two different positions $\left(C_{s}-\ln n_{a}\right.$ and $\left.C_{s}-n_{b}\right)$, and the interstitial carbon into the lattice of $\ln _{2} O_{3}\left(C_{i}\right)$. Specific properties, formation energies, bond lengths and ionic valence, were listed in Table S2. The results revealed the interstitial carbon model had the smallest formation energy of $7.59 \mathrm{eV}$ (Figure 1d) and required minimal energy injection than the other three cases. In comparison, model III, the substitution of indium at position B, had the second lowest formation energy. However, the atomic radii of carbon and indium atoms were too far apart for substitution doping to occur ${ }^{31}$. Therefore, we speculated the carbon atom tended to be an interstitial state to anchor into the $\ln _{2} \mathrm{O}_{3}$ lattice. Interstitial carbon could further affect the coordination of neighboring atoms to manipulate electronic properties. As seen from the X-ray absorption fine structure (XAFS), the In K-edge $k^{2} \chi(k)$ vibrational lines presented a great difference between $\mathrm{C}-\mathrm{In}_{2} \mathrm{O}_{3-x}$ and standard cubic $\ln _{2} \mathrm{O}_{3}$, which qualitatively revealed distinct local atomic arrangements (inset of Figure 1e). In Figure 1e, the four main peaks could be regarded as In-In shell and In-O shell in the R space for refined local atomic arrangements. We found that doped carbon atoms shortened the length of In-In bonds and enlarged the length of In-O bonds (Table S3). In addition, the maximum K-edge absorption of C- $\mathrm{In}_{2} \mathrm{O}_{3}$ was reduced to $27938.7 \mathrm{eV}$ (Figure S4), indicating the generation of oxygen vacancy, the coordination number reduction of In-O and In-In, which resulted in higher disorder degrees ${ }^{32,33}$. Compared with standard cubic $\ln _{2} \mathrm{O}_{3}$, the $\mathrm{C}-\mathrm{In}_{2} \mathrm{O}_{3}$ with the presence of a noticeable structure distortion helped to maintain structural stability ${ }^{34,35}$.

Raman spectra provided more proof on local structural change and distortion of C- $\ln _{2} \mathrm{O}_{3-\mathrm{x}}$ (Figure S5). Distinct photon modes at $309,407,491$, and $628 \mathrm{~cm}^{-1}$ were consistent with Raman active modes of the cubic structure $\ln _{2} \mathrm{O}_{3}{ }^{36,37}$, but when the carbon was doped into the $\ln _{2} \mathrm{O}_{3}$ lattice with different carbon doping levels, these highlighted peaks become smoother, suggesting introduced carbon disrupts original lattice structure ${ }^{38}$. To investigate the effect of doped carbon on the chemical state and electronic structure of $\ln _{2} \mathrm{O}_{3}$, we performed X-ray photoelectron spectroscopy (XPS) characterization. From the In 3d core-level spectra (Figure 1f), the two peaks located at $443.7 \mathrm{eV}$ and $451.4 \mathrm{eV}$ were assigned to the $\ln 3 \mathrm{~d}_{5 / 2}$ and $\ln 3 \mathrm{~d}_{3 / 2}$ signals ${ }^{39,40}$. However, with interference from carbon, both peaks moved toward higher binding energy with an about $0.5 \mathrm{eV}$ shift, confirming indium atoms did accept electrons from doped carbon. To prove the XPS results, we computed the charge difference density (isosurface value is $3 \mathrm{e} \mathrm{nm}^{-3}$ ) of model IV in Figure 1d and found much net gain of charge was distributed around indium atom (inset of Figure 1f). Experimental data corroborated theoretical calculation, proving that carbon was successfully anchored in the lattice of $\ln _{2} \mathrm{O}_{3}$ and therefore altered its original electronic structure. 


\section{Photothermal $\mathrm{CO}_{2}$ reduction performance}

To reveal the advantages of $\mathrm{C}-\mathrm{In}_{2} \mathrm{O}_{3}$ in the photothermal-driven RWGS reaction, we conducted the catalytic performance tests

(Figure 2a) of the $\mathrm{V}_{0}$-poor $\ln _{2} \mathrm{O}_{3}, \mathrm{~V}_{0}$-rich $\ln _{2} \mathrm{O}_{3-x}$, and $\mathrm{C}-\mathrm{In}_{2} \mathrm{O}_{3-x}$ with different carbon doping levels (Table S4). Benefiting from the richer concentration of active sites, $\mathrm{V}_{\mathrm{o}}$-rich $\ln _{2} \mathrm{O}_{3-\mathrm{x}}$ had $18.2 \mathrm{mmol} \mathrm{g}_{\mathrm{cat}}{ }^{-1} \mathrm{~h}^{-1}$ of $\mathrm{CO}$ production rate, which was three times higher than that of $\mathrm{V}_{\mathrm{o}}$-poor $\ln _{2} \mathrm{O}_{3}$. This result was consistent with the literature analyses that oxygen vacancy facilitated $\mathrm{CO}_{2}$ adsorption to yield high RWGS reactivity ${ }^{18,23,41}$. When the appropriate amount of carbon was doped, the catalytic activity of $C-\ln _{2} \mathrm{O}_{3-x^{-3}}$ achieved six-fold enlargement compared with $\mathrm{V}_{\mathrm{o}}$-rich $\mathrm{In}_{2} \mathrm{O}_{3-\mathrm{x}}\left(123.6 \mathrm{mmol} \mathrm{g}_{\mathrm{cat}}{ }^{-1} \mathrm{~h}^{-1}\right)$. Low doping concentration limited light absorption capacity to enhance the photothermal conversion efficiency in endothermic RWGS reaction while high doping concentration led that extra carbon cannot anchor in the $\ln _{2} \mathrm{O}_{3}$ lattice and form inert "free carbon" around the $\ln _{2} \mathrm{O}_{3}$ nanoparticle to compete for the light energy. The more detail of the mechanism for the high activity will be discussed in next section. With the optimal sample, the $\mathrm{CO}_{2}$ conversion rate of $\mathrm{C}-\mathrm{In}_{2} \mathrm{O}_{3-\mathrm{x}}$ eventually reached $45 \%$ with near-unity $\mathrm{CO}$ selectivity without the production of $\mathrm{CH}_{4}$ (Figure $2 \mathrm{~b}$ ) and the remarkable catalytic performance kept holding beyond 20 cycles in stability test (Figure 2c). The blank control test under the photothermal conditions also confirmed the doped carbon in the $\mathrm{C}-\mathrm{In}_{2} \mathrm{O}_{3-\mathrm{x}}$ was stable and could not be decomposed to $\mathrm{CO}_{2}$ or $\mathrm{CO}$ (Figure S6). Further, the turnover number of the cyclic stability test (the amount of yield product/the amount of catalyst) was 190.56 that was much larger than 1, indicating photothermal RWGS reaction exhibits typical feature of catalysis.

\section{Mechanism and pathway of photothermal $\mathrm{CO}_{2}$ reduction}

Photothermal catalysis of $\mathrm{CO}_{2}$ reduction involves two main steps. The first step is light absorption and photothermal transformation via the active sites on the catalyst surface (local temperature effect); the second is $\mathrm{CO}_{2}$ adsorption and subsequent catalytic process ${ }^{28}$. In the first step, the catalysts are required to possess broader spectral coverage to maximize energy absorption and energy transfer efficiency. Because the photoexcited carriers (electron-hole pairs) could heat the nonplasmonic $\mathrm{C}-\mathrm{In}_{2} \mathrm{O}_{3-\mathrm{x}}$ through non-radiative recombination from either Auger or Shockley-Read-Hall (i.e., trap assisted) to drive the thermally catalytic process, which rendered the mechanism for lattice vibrations and heat generation ${ }^{6,42}$, and powerfully assisted in lowering the photochemical activation energy ${ }^{43,44}$. Unlike the pale yellow of $\ln _{2} \mathrm{O}_{3}$ crystals or the pale gray of $\ln _{2} \mathrm{O}_{3-x}$, the color of $\mathrm{C}-\mathrm{In}_{2} \mathrm{O}_{3-\mathrm{x}}$ appeared jet black (inset of Figure 3a), having broader photon absorption ranges from 250 to $2400 \mathrm{~nm}$ and stronger absorption intensity. Doped carbon atoms could extend the scope to the visible light region and defect state caused by oxygen vacancy further extended it to infrared region (Figure 3a). Combined with the bandgap structure (Figure S7) and ultraviolet photoelectron spectroscopy (UPS) (Figure S8), a schematic illustration of the bandgap structure of C- $\ln _{2} \mathrm{O}_{3-\mathrm{x}}$ was depicted and shown in Figure S9. The band structure and density of states (DOS) of C- $-\mathrm{In}_{2} \mathrm{O}_{3-\mathrm{x}}$ regulated by doped carbon could be explained using density functional theory (DFT). In Figure S10, compared with the cubic $\ln _{2} \mathrm{O}_{3}$, when an oxygen vacancy was generated around an interstitial carbon, a new defect energy level with more massive DOS emerges. In this case, the electrons can be easily excited into the conduction band through the intermediate state, thereby achieving higher photoconversion efficiency ${ }^{45-47}$.

On the other hand, the radiative recombination competed against non-radiative recombination. After carbon doping, the fluorescence significantly quenched, demonstrating that radiative recombination of the photon-generated carriers had been suppressed by extracting the electrons (Figure S11). Therefore, the $\mathrm{C}-\mathrm{In}_{2} \mathrm{O}_{3-\mathrm{x}}$ system could reach near $400{ }^{\circ} \mathrm{C}$ within 10 minutes, which is far higher temperature than those of $\mathrm{V}_{0}$-poor $\ln _{2} \mathrm{O}_{3}$ and $\mathrm{V}_{\mathrm{o}}$-rich $\ln _{2} \mathrm{O}_{3-\mathrm{x}}$ (Figure 3b). The surface of $\mathrm{C}-\ln _{2} \mathrm{O}_{3-\mathrm{x}}$ worked as a "nanoscale heat source," which absorbed the photon energy and rapidly converted it into heat that was conducted to the active sites of $\mathrm{C}-\ln _{2} \mathrm{O}_{3-\mathrm{x}}$ in a short time. 
The next step was adsorption of gas molecules onto the surface and subsequent catalytic process. For clarity, in temperatureprogrammed desorption (TPD) of $\mathrm{CO}_{2}$, the peak at $480^{\circ} \mathrm{C}$ indicated the adsorption was in the form of chemisorption via the oxygen vacancies on $\mathrm{C}-\ln _{2} \mathrm{O}_{3-x} 48,49$. If the oxygen vacancy is filled under annealing in air, the adsorption capacity could be notably reduced (Figure S12). In order to prove the role of oxygen vacancy as the active site was in the catalytic process, we conducted comparative DFT calculations of $\operatorname{In}_{2} \mathrm{O}_{3}$ and $\mathrm{In}_{2} \mathrm{O}_{3}-\mathrm{O}_{\text {vac }}$ [110] slabs (Figure S13 -15). The Gibbs free energy for the intermediate ${ }^{*} \mathrm{CO}$ of RWGS reaction on $\ln _{2} \mathrm{O}_{3}$ slab without oxygen vacancy was $0.66 \mathrm{eV}$ which was higher than the slab with oxygen vacancy $(0.32$ $\mathrm{eV}$ ). Thus, a high concentration of oxygen vacancies was expected to increase the capacity of $\mathrm{CO}_{2}$ adsorption and lower the energy barrier of photothermal $\mathrm{CO}_{2}$ reduction RWGS reaction. Electron spin resonance spectroscopy (ESR) effectively evaluated the oxygen vacancy concentration (Figure $3 c$ ). Limited signals of $\mathrm{V}_{\mathrm{o}}$-poor $\ln _{2} \mathrm{O}_{3}$ and $\mathrm{V}_{\mathrm{o}}$-rich $\ln _{2} \mathrm{O}_{3-\mathrm{x}}$ were detected, while a stronger signal of $\mathrm{C}-\mathrm{In}_{2} \mathrm{O}_{3-\mathrm{x}}$ appeared at $\mathrm{g}=2.004$, which could be explained as more concentrated electrons are trapped around the surface oxygen vacancies ${ }^{50}$. Next, to prove the carbon doping has a function in elevating oxygen vacancy concentration, the formation energy of oxygen vacancy was simulated (inset of Figure 3c). In non-defective $\ln _{2} \mathrm{O}_{3}$ crystal, the oxygen vacancy formation energy was $4.98 \mathrm{eV}$, while the energy decreased to $3.10 \mathrm{eV}$ after interstitial carbon anchored into $\ln _{2} \mathrm{O}_{3}$ lattice (Table S3). Our result suggested that interstitial carbon assembled with oxygen vacancy to form a higher concentration active sites, thereby pushing forward the RWGS reaction.

We then performed the in-situ Fourier Transform Infrared (FTIR) characterization in a flow cell to investigate the catalytic pathway of RWGS reaction on the active sites of $\mathrm{C}-\mathrm{In}_{2} \mathrm{O}_{3-\mathrm{x}}$. As displayed in Figure $3 \mathrm{~d}$, the $\mathrm{C}-\mathrm{In}_{2} \mathrm{O}_{3-\mathrm{x}}$ exhibited $\mathrm{OH}$ stretching band within the range $3400-3730 \mathrm{~cm}^{-1}$. Peaks located at 1500 and $1390 \mathrm{~cm}^{-1}$ were identified as chemisorbed $\mathrm{CO}_{2}$ and $\mathrm{H}_{2}$ molecules, as well as bicarbonate $\left(\mathrm{HCO}_{3}{ }^{-}\right)$and carbonate $\left(\mathrm{CO}_{3}^{-}\right)$formation. The detectable peak at $2894 \mathrm{~cm}^{-1}$ was attributed to bending vibration $\delta(\mathrm{CH})$. Here, the peaks corresponding to crucial intermediate methoxy $\left(\mathrm{H}_{3} \mathrm{CO} \mathrm{O}^{*}\right)$ in the spectra were at 2839 and $1090 \mathrm{~cm}^{-1}$, and $\mathrm{COOH}^{*}$ were at 1578 and $1232 \mathrm{~cm}^{-1}$. Therefore, the incoming electrons coupled with resident protons reduced the amount of $\mathrm{CO}_{2}$ molecules at the active sites, oxygen vacancy, then formed $\mathrm{COOH}^{*}$ and $\mathrm{H}_{3} \mathrm{CO}$ * intermediates and transferred into $\mathrm{CO}^{*}$ consistently. Simultaneously, the peak at $2107 \mathrm{~cm}^{-1}$ and $1643 \mathrm{~cm}^{-1}$ were the diagnostic vibrational modes of $\mathrm{CO}$ and $\mathrm{H}_{2} \mathrm{O}$ respectively, indicating the reaction pathway that features $\mathrm{CO}^{*}$ as the intermediate from carboxyl through the RWGS reaction ${ }^{51-54}$. In general, oxygen vacancy with doped carbon as active sites effectively activated $\mathrm{CO}_{2}$ molecules and stabilized the intermediates, so as to reduce the activation energy and promote photothermal $\mathrm{CO}_{2}$ reduction (Figure 3e).

\section{Discussion}

In summary, photothermal catalytic $\mathrm{CO}_{2}$ reduction is a promising technology to alter the traditional thermo-catalytic reaction, which thermodynamically improves activity under light conditions without additional energy input. The dual-function carbon doping is a feasible option to enhance and stabilize photothermal catalyst activity of $\ln _{2} \mathrm{O}_{3}$. Doped carbon is more conducive to the light absorption and the photothermal conversion efficiency improvement; benefiting from formation energy reduction of oxygen vacancy, high concentration active sites are obtainable. As a result, the $\mathrm{C}-\mathrm{In}_{2} \mathrm{O}_{3-\mathrm{x}}$ has $123.6 \mathrm{mmol} \mathrm{g}^{-1} \mathrm{~h}^{-1}$ of $\mathrm{CO}$ production rates and nearly $100 \%$ selectivity. This work demonstrates that the microstructure modulation of crystal defect in catalyst can significantly enhanced the utilization of solar light and then to drive efficient and stable catalytic reaction, which achieves highperformance solar-to-chemical energy conversion.

\section{Experimental Section}

Materials: Synthesis of $\mathrm{V}_{\mathrm{o}}$-poor $\ln _{2} \mathrm{O}_{3}$ and $\mathrm{V}_{\mathrm{o}}$-rich $\ln _{2} \mathrm{O}_{3-\mathrm{x}}$ nano partials: First, $1 \mathrm{mmol}$ indium nitrate was added into $60 \mathrm{~mL}$ deionized water, magnetically stirred for $20 \mathrm{~min}$, then mixed with $3 \mathrm{mmol}$ urea followed by another $10 \mathrm{~min}$ stirring. After, the mixture was transferred into a $100 \mathrm{~mL}$ Teflon-lined stainless-steel autoclave, sealed and maintained at $140^{\circ} \mathrm{C}$ for 12 hours. The whole system naturally cools down to room temperature. After centrifuging the mixture, the final product was collected, washed with distilled water and ethanol several times and then dried in air at $60^{\circ} \mathrm{C}$. Calcination is at $600{ }^{\circ} \mathrm{C}$ for $2 \mathrm{~h}$ in air and $\mathrm{H}_{2}-\mathrm{Ar}$ with a heating rate of $5^{\circ} \mathrm{C} \mathrm{min}-1$ to fabricate the $\mathrm{V}_{\mathrm{o}}$-poor $\ln _{2} \mathrm{O}_{3}$ and $\mathrm{V}_{\mathrm{o}}$-rich $\ln _{2} \mathrm{O}_{3-\mathrm{x}}$. The synthesis procedure for $\mathrm{C}-\mathrm{In}_{2} \mathrm{O}_{3}$ is similar to that 
of $\mathrm{V}_{\mathrm{o}}$-poor $\mathrm{In}_{2} \mathrm{O}_{3}$ and $\mathrm{V}_{\mathrm{o}}$-rich $\ln _{2} \mathrm{O}_{3-\mathrm{x}}$, except that $1 \mathrm{mmol}$ of indium nitrate, $3 \mathrm{mmol}$ urea and different amounts of glucose $(0.1,0.2$, $0.3,0.4$, and $0.5 \mathrm{~g}$ ) were simultaneously added into the starting solution for hydrothermal synthesis, and calcination at $600{ }^{\circ} \mathrm{C}$ for $2 \mathrm{~h}$ in $\mathrm{Ar}$.

Reaction condition and activity evaluation: Photothermal $\mathrm{CO}_{2}$ conversion by $\mathrm{H}_{2}$ was carried out in a batch type reaction system with a total volume of $330 \mathrm{ml}$. After evacuation of reaction system, $\mathrm{CO}_{2}$ and $\mathrm{H}_{2}$ (the molar ratio of $\mathrm{H}_{2}$ to $\mathrm{CO}_{2}$ is $1: 1$ ) were injected. A 300W Xe lamp was used as irradiation source to drive the photothermal $\mathrm{CO}_{2}$ conversion. The light intensity is $2.98 \mathrm{~W} \mathrm{~cm}{ }^{-2}$. The temperature of catalyst surface is measured by the infrared thermometer, and the reflectance coefficient is 0.78 . For all experiments, $0.05 \mathrm{~g}$ of sample was used and spread onto a round shape air-permeable quartz fiber filter with the area of $7 \mathrm{~cm}^{2}$. The quartz fiber filter film is fixed on the stage inside the reactor. The tip of thermometer was maintained an intimate contact with the sample (thickness of catalyst powder is about 1 2 mm). The contents of $\mathrm{CO}_{2}$ and $\mathrm{CO}$ in the reaction system were sampled and measured with a gas chromatograph (GC-2014, Shimadzu) equipped with a methanizer and flame ionization detector according to the standard curves.

Sample characterization: XRD patterns were recorded on an X-Pert diffractometer equipped with graphite monochromatized Cu-K radiation. The morphologies were characterized by field-emission SEM (Hitachi, s4800) and TEM (JEOL, 2100F). The diffuse reflection spectra of catalysts were measured by UV-vis-NIR spectrophotometer (Shimadzu, UV-3600) from 220 to $2400 \mathrm{~nm}$. Chemical valence of the surface was analyzed by XPS (Escalab 250Xi, Thermo Scientific, America). The PL properties of materials were investigated by PL spectra (Horiba Fluorolog-3) with the excitation light wavelength of $350 \mathrm{~nm}$. The ESR spectra measurements were executed on JES-FA200 X-band spectrometer. The catalyst temperature was probed by a digital thermometer (Custom, CT-1200D). Raman spectra were recorded by Horiba (Xplora Plus) instrument. The pore size distribution was determined with a surface area analyzer (BEL Sorp-II mini, BEL Japan Co., Japan) by the BET method. The FTIR spectrum was executed on Nicolet 6700. In situ FTIR spectra have been recorded by a Nicolet 6700 Fourier transform instrument, using conventional IR cells connected to a gas manipulation apparatus. XAS measurement for the In K-edge was performed in fluorescence mode on beamline 20-BM-B with electron energy of $7 \mathrm{GeV}$ and an average current of $100 \mathrm{~mA}$ which is located in the Advanced Photon Source at Argonne National Laboratory.

\section{Declarations}

Acknowledgements

This work received financial support from the National Natural Science Foundation of China (Grants 21972052 and 21633004) and JSPS KAKENHI of Japan (Grant Number JP18H02065).

\section{References}

1. Walter, M.G. et al. Solar Water Splitting Cells. Chem Rev 110, 6446-6473 (2010).

2. Chu, S. \& Majumdar, A. Opportunities and challenges for a sustainable energy future. Nature 488, 294-303 (2012).

3. Dusastre, V. \& Martiradonna, L. Materials for sustainable energy. Nat Mater 16, 15-15 (2017).

4. Brockway, P.E., Owen, A., Brand-Correa, L.I. \& Hardt, L. Estimation of global final-stage energy-return-on-investment for fossil fuels with comparison to renewable energy sources. Nature Energy 4, 612-621 (2019).

5. Shindell, D. \& Smith, C.J. Climate and air-quality benefits of a realistic phase-out of fossil fuels. Nature 573, $408-411$ (2019).

6. Ghoussoub, M., Xia, M., Duchesne, P.N., Segal, D. \& Ozin, G. Principles of photothermal gas-phase heterogeneous $\mathrm{CO}_{2}$ catalysis. Energ Environ Sci 12, 1122-1142 (2019).

7. Li, Z.H. et al. Co-Based Catalysts Derived from Layered-Double-Hydroxide Nanosheets for the Photothermal Production of Light Olefins. Adv Mater 30, 1800527 (2018). 
8. Ren, J. et al. Targeting Activation of $\mathrm{CO}_{2}$ and $\mathrm{H}_{2}$ over Ru-Loaded Ultrathin Layered Double Hydroxides to Achieve Efficient Photothermal $\mathrm{CO}_{2}$ Methanation in Flow-Type System. Adv Energy Mater 7, 1601657 (2017).

9. Zhao, L. et al. Solar-Driven Water-Gas Shift Reaction over $\mathrm{CuO}_{\mathrm{x}} / \mathrm{Al}_{2} \mathrm{O}_{3}$ with $1.1 \%$ of Light-to-Energy Storage. Angew Chem Int Ed Eng/ 58, 7708-7712 (2019).

10. Hoegh-Guldberg, O. et al. The human imperative of stabilizing global climate change at 1.5 degrees C. Science 365,1263 (2019).

11. Liu, C., Colon, B.C., Ziesack, M., Silver, P.A. \& Nocera, D.G. Water splitting-biosynthetic system with $\mathrm{CO}_{2}$ reduction efficiencies exceeding photosynthesis. Science 352, 1210-1213 (2016).

12. Asadi, M. et al. Nanostructured transition metal dichalcogenide electrocatalysts for $\mathrm{CO}_{2}$ reduction in ionic liquid. Science $\mathbf{3 5 3}$, 467-470 (2016).

13. Ellis, P.R., Enache, D.I., James, D.W., Jones, D.S. \& Kelly, G.J. A robust and precious metal-free high performance cobalt Fischer-Tropsch catalyst. Nat Catal 2, 623-631 (2019).

14. Zhu, C., Liu, J., Li, M.B. \& Backvall, J.E. Palladium-catalyzed oxidative dehydrogenative carbonylation reactions using carbon monoxide and mechanistic overviews. Chem Soc Rev 49, 341-353 (2019).

15. Jouny, M. et al. Formation of carbon-nitrogen bonds in carbon monoxide electrolysis. Nat Chem 11, 846-851 (2019).

16. Kinney, R.G. \& Arndtsen, B.A. Decarboxylation with Carbon Monoxide: The Direct Conversion of Carboxylic Acids into Potent Acid Triflate Electrophiles. Angew Chem Int Ed Eng/ 58, 5085-5089 (2019).

17. Morris, P.J.T. The Legacy of Mond,Ludwig. Endeavour 13, 34-40 (1989).

18. Hou, J. et al. Atomically Thin Mesoporous $\ln _{2} \mathrm{O}_{3-x} / \ln _{2} \mathrm{~S}_{3}$ Lateral Heterostructures Enabling Robust Broadband-Light PhotoElectrochemical Water Splitting. Adv Energy Mater 8, 1701114 (2018).

19. Chao, Y. et al. Ultrathin Visible-Light-Driven Mo Incorporating $\ln _{2} \mathrm{O}_{3}-Z n \ln _{2} \mathrm{Se}_{4} Z$ Z-Scheme Nanosheet Photocatalysts. Adv Mater 31, e1807226 (2019).

20. Wang, L. et al. Black indium oxide a photothermal CO2 hydrogenation catalyst. Nat Commun 11 (2020).

21. Rui, N. et al. $\mathrm{CO}_{2}$ hydrogenation to methanol over $\mathrm{Pd} / \mathrm{In}_{2} \mathrm{O}_{3}$ : effects of $\mathrm{Pd}$ and oxygen vacancy. Applied Catalysis $B$ : Environmental 218, 488-497 (2017).

22. Hoch, L.B. et al. Nanostructured Indium Oxide Coated Silicon Nanowire Arrays: A Hybrid Photothermal/Photochemical Approach to Solar Fuels. Acs Nano 10, 9017-9025 (2016).

23. Ye, J., Liu, C., Mei, D. \& Ge, Q. Active Oxygen Vacancy Site for Methanol Synthesis from $\mathrm{CO}_{2}$ Hydrogenation on $\operatorname{In}_{2} \mathrm{O}_{3}(110)$ : $A$ DFT Study. Acs Cata/3, 1296-1306 (2013).

24. Gao, P. et al. Direct conversion of $\mathrm{CO}_{2}$ into liquid fuels with high selectivity over a bifunctional catalyst. Nat Chem 9, 10191024 (2017).

25. Ha, M. et al. Multicomponent Plasmonic Nanoparticles: From Heterostructured Nanoparticles to Colloidal Composite Nanostructures. Chem Rev 119, 12208-12278 (2019).

26. Prieto, G. et al. Hollow Nano- and Microstructures as Catalysts. Chem Rev 116, 14056-14119 (2016).

27. Cao, X., Tan, C., Sindoro, M. \& Zhang, H. Hybrid micro-/nano-structures derived from metal-organic frameworks: preparation and applications in energy storage and conversion. Chem Soc Rev 46, 2660-2677 (2017).

28. Qi, Y. et al. Photoinduced Defect Engineering: Enhanced Photothermal Catalytic Performance of 2D Black $\operatorname{In}_{2} \mathrm{O}_{3-\mathrm{x}} \mathrm{Nanosheets}$ with Bifunctional Oxygen Vacancies. Adv Mater 32, 1903915 (2019).

29. Li, L. et al. Insight into the Effect of Oxygen Vacancy Concentration on the Catalytic Performance of $\mathrm{MnO}_{2}$. Acs Catal 5, 48254832 (2015)..

30. Cui, Y.L.S. et al. Refilling Nitrogen to Oxygen Vacancies in Ultrafine Tungsten Oxide Clusters for Superior Lithium Storage. Adv Energy Mater 9, 1902148 (2019).

31. Yu, W., Zhang, J. \& Peng, T. New insight into the enhanced photocatalytic activity of N-, C- and S-doped ZnO photocatalysts. Applied Catalysis B: Environmental 181, 220-227 (2016). 
32. Zhao, Y. et al. Defect-Rich Ultrathin ZnAl-Layered Double Hydroxide Nanosheets for Efficient Photoreduction of $\mathrm{CO}_{2}$ to $\mathrm{CO}$ with Water. Adv Mater 27, 7824-7831 (2015).

33. Haneda, M., Kintaichi, Y., Bion, N. \& Hamada, H. Mechanistic study of the effect of coexisting $\mathrm{H}_{2} \mathrm{O}$ on the selective reduction of $\mathrm{NO}$ with propene over sol-gel prepared $\mathrm{In}_{2} \mathrm{O}_{3}-\mathrm{Al}_{2} \mathrm{O}_{3}$ catalyst. Applied Catalysis B: Environmental 42, 57-68 (2003).

34. Sun, Y., Gao, S., Lei, F. \& Xie, Y. Atomically-thin two-dimensional sheets for understanding active sites in catalysis. Chem Soc $\operatorname{Rev}$ 44, 623-636 (2015).

35. Sun, Y. et al. Fabrication of flexible and freestanding zinc chalcogenide single layers. Nat Commun 3, 1057 (2012).

36. Kranert, C., Schmidt-Grund, R. \& Grundmann, M. Raman active phonon modes of cubic $\ln _{2} \mathrm{O}_{3}$. physica status solidi (RRL) Rapid Research Letters 8, 554-559 (2014).

37. Yin, W.Y. et al. $\ln (\mathrm{OH})_{3}$ and $\ln _{2} \mathrm{O}_{3}$ Micro/Nanostructures: Controllable NaOAc-Assisted Microemulsion Synthesis and Raman Properties. J Phys Chem C113, 19493-19499 (2009).

38. Gu, F.B., Li, C.J., Han, D.M. \& Wang, Z.H. Manipulating the Defect Structure (V-O) of $\ln _{2} \mathrm{O}_{3}$ Nanoparticles for Enhancement of Formaldehyde Detection. Acs App/ Mater Inter 10, 933-942 (2018).

39. Cao, S.-W. et al. Solar-to-fuels conversion over $\mathrm{In}_{2} \mathrm{O}_{3} / \mathrm{g}-\mathrm{C}_{3} \mathrm{~N}_{4}$ hybrid photocatalysts. Applied Catalysis B: Environmental 147, 940-946 (2014).

40. Prim, A. et al. A Novel Mesoporous CaO-Loaded In203 Material for $\mathrm{CO}_{2}$ Sensing. Advanced Functional Materials 17, $2957-$ 2963 (2007).

41. Ye, J., Liu, C. \& Ge, Q. DFT Study of $\mathrm{CO}_{2}$ Adsorption and Hydrogenation on the In2O3 Surface. The Journal of Physical Chemistry C116, 7817-7825 (2012).

42. Hoch, L.B. et al. Carrier dynamics and the role of surface defects: Designing a photocatalyst for gas-phase $\mathrm{CO}_{2}$ reduction. Proc Natl Acad Sci 113, 8011-8020 (2016).

43. He, L. et al. Spatial Separation of Charge Carriers in $\ln _{2} \mathrm{O}_{3-\mathrm{x}}(\mathrm{OH})_{y}$ Nanocrystal Superstructures for Enhanced Gas-Phase Photocatalytic Activity. Acs Nano 10, 5578-5586 (2016).

44. Ghuman, K.K. et al. Illuminating $\mathrm{CO}_{2}$ reduction on frustrated Lewis pair surfaces: investigating the role of surface hydroxides and oxygen vacancies on nanocrystalline $\mathrm{In}_{2} \mathrm{O}_{3-\mathrm{x}}(\mathrm{OH})_{\mathrm{y}}$. Phys Chem Chem Phys 17, 14623-14635 (2015).

45. Yang, M.Q. et al. Disorder Engineering in Monolayer Nanosheets Enabling Photothermic Catalysis for Full Solar Spectrum (250-2500 nm) Harvesting. Angew Chem Int Edit 58, 3077-3081 (2019).

46. Rahman, M.A. et al. Defect-rich decorated $\mathrm{TiO}_{2}$ nanowires for super-efficient photoelectrochemical water splitting driven by visible light. Energ Environ Sci 8, 3363-3373 (2015).

47. Nowotny, J. et al. Defect chemistry and defect engineering of $\mathrm{TiO}_{2}$-based semiconductors for solar energy conversion. Chem Soc Rev 44, 8424-8442 (2015).

48. Rui, N. et al. $\mathrm{CO}_{2}$ hydrogenation to methanol over $\mathrm{Pd} / \mathrm{In}_{2} \mathrm{O}_{3}$ : effects of Pd and oxygen vacancy. Appl Catal B-Environ 218, 488497 (2017).

49. Martin, O. et al. Indium Oxide as a Superior Catalyst for Methanol Synthesis by $\mathrm{CO}_{2}$ Hydrogenation. Angew Chem Int Ed Engl 55, 6261-6265 (2016).

50. Lei, F. et al. Oxygen Vacancies Confined in Ultrathin Indium Oxide Porous Sheets for Promoted Visible-Light Water Splitting. $J$ Am Chem Soc 136, 6826-6829 (2014).

51. Yan, T. et al. Polymorph selection towards photocatalytic gaseous $\mathrm{CO}_{2}$ hydrogenation. Nat Commun 10, 1-10 (2019).

52. Chen, G. et al. From Solar Energy to Fuels: Recent Advances in Light-Driven C1 Chemistry. Angew Chem Int Ed Eng/ 58, 1752817551 (2019).

53. Sun, Q., Liu, C.W., Pan, W., Zhu, Q.M. \& Deng, J.F. In situ IR studies on the mechanism of methanol synthesis over an ultrafine $\mathrm{Cu} / \mathrm{ZnO} / \mathrm{Al}_{2} \mathrm{O}_{3}$ catalyst. App/ Catal a-Gen 171, 301-308 (1998).

54. Choi, S. et al. Catalytic behavior of metal catalysts in high-temperature RWGS reaction: In-situ FT-IR experiments and firstprinciples calculations. Sci Rep 7, 41207 (2017). 


\section{Scheme}

Scheme 1 is available in the Supplementary Files.

\section{Figures}
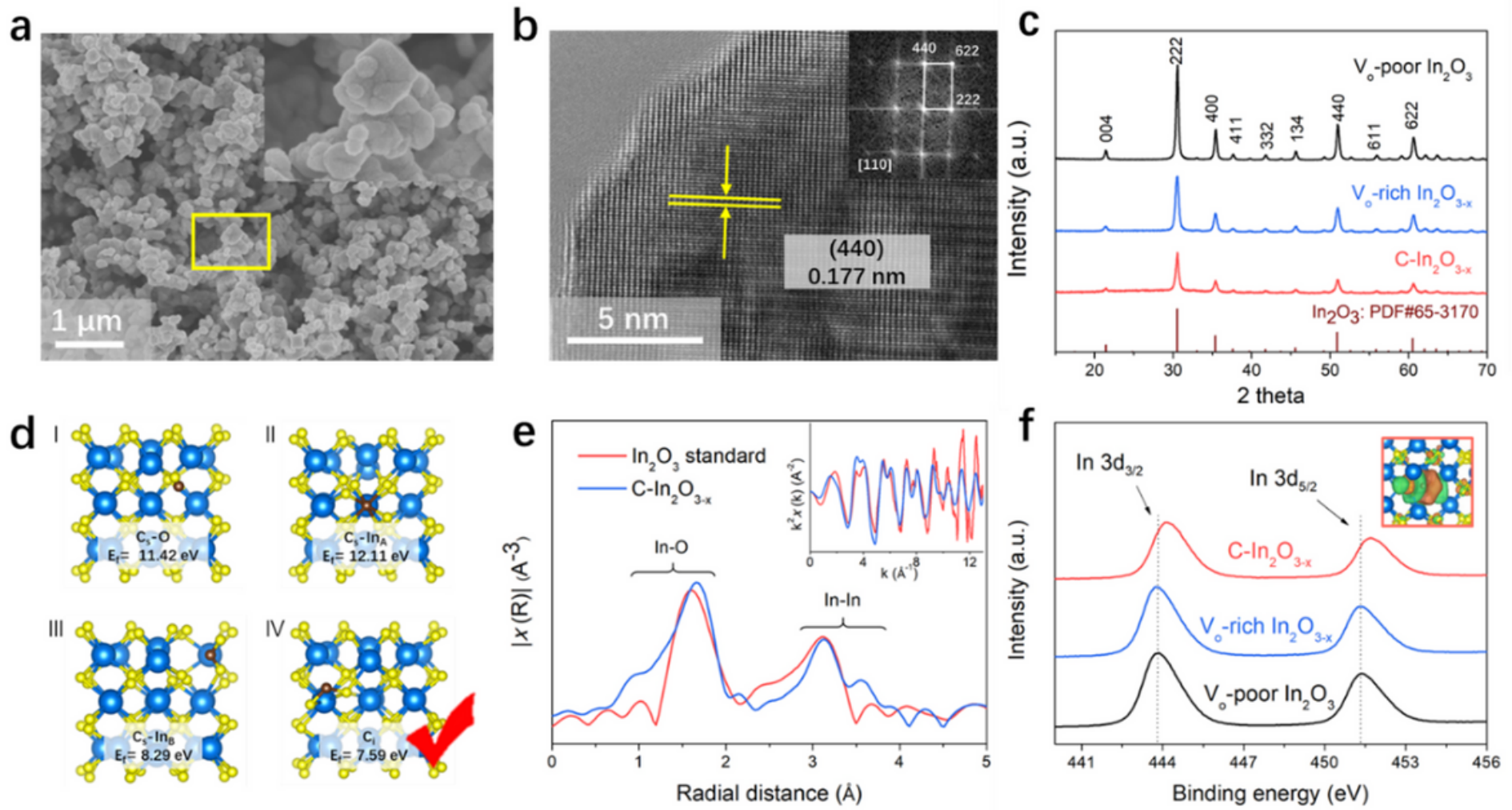

\section{Figure 1}

(a) SEM of C-In2O3-x, inset: the part of the region enclosed by the yellow square, (b) HR-TEM image of C-In2O3-x, inset: The Fast Fourier transform corresponding yellow square, (c) XRD patterns of Vo-poor In2O3, Vo-rich In2O3-x and C-In203-x, (d) Formation energy of Cs-O, Cs-Ina, Cs-Inb and Ci, (e) In K-edge extended XAFS oscillation function k2 $\chi(\mathrm{k})$, Inset: the corresponding Fouriertransformed data for standard In2O3 and C-In203-x, (f) XPS spectra of the In 3d core level peak regions of C-In2O3-x, inset: charge difference density of In2O3 with one $C$ atom interstitial with one $\mathrm{O}$ atom vacancy. 
a

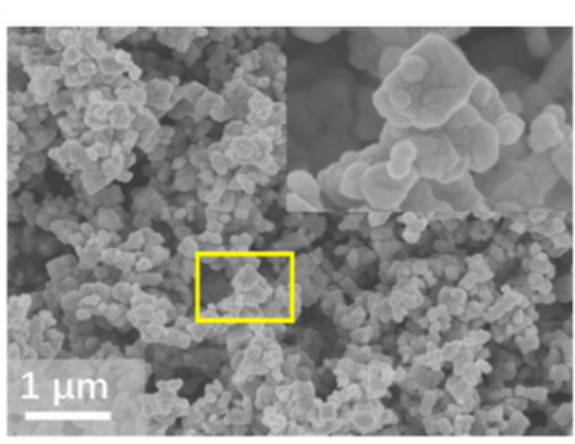

d 1
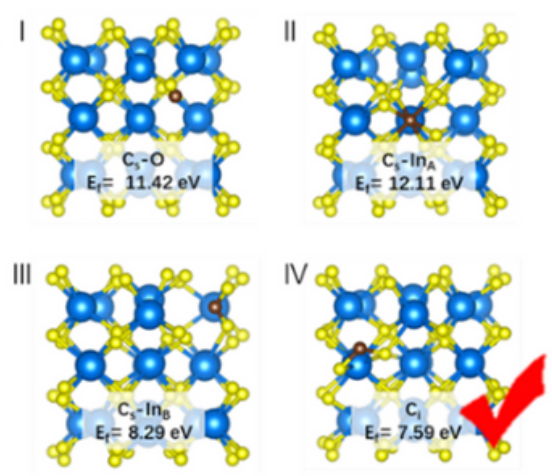

b

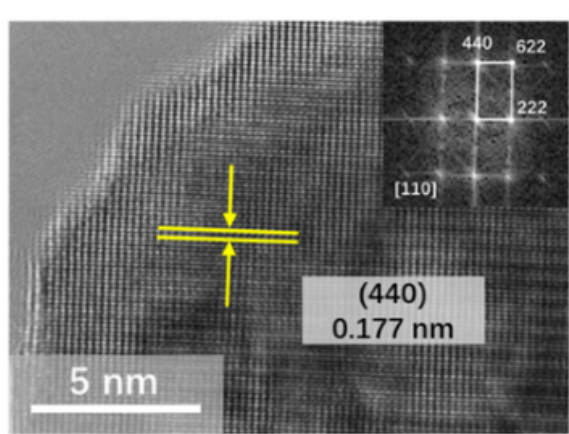

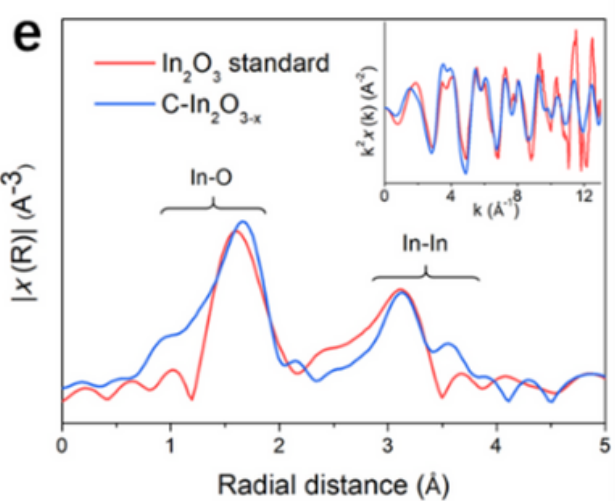
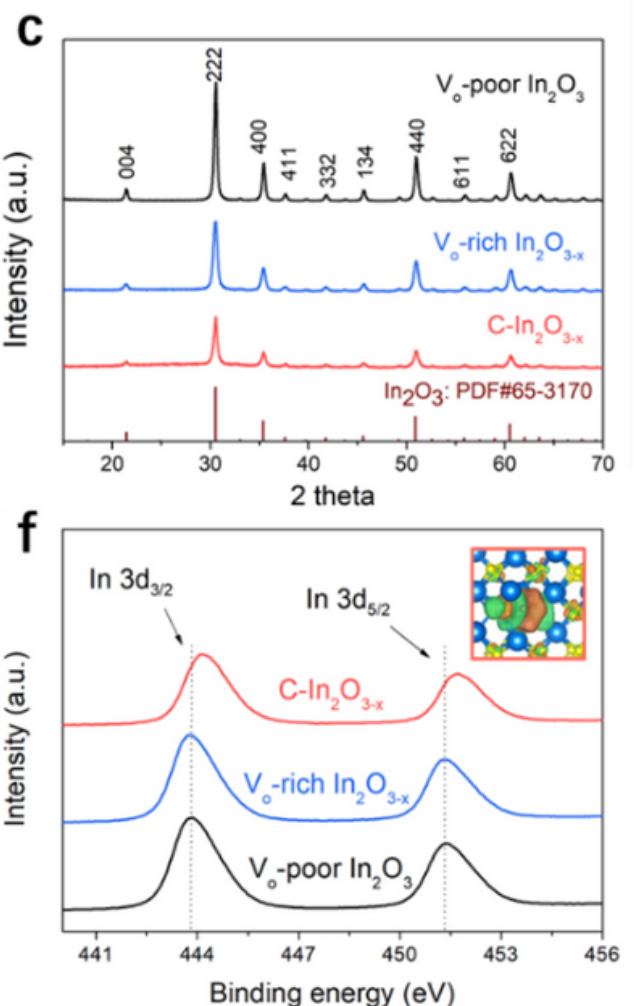

\section{Figure 1}

(a) SEM of C-In203-x, inset: the part of the region enclosed by the yellow square, (b) HR-TEM image of C-In2O3-x, inset: The Fast Fourier transform corresponding yellow square, (c) XRD patterns of Vo-poor In203, Vo-rich In203-x and C-In2O3-x, (d) Formation energy of Cs-O, Cs-Ina, Cs-Inb and Ci, (e) In K-edge extended XAFS oscillation function k2 $x(\mathrm{k})$, Inset: the corresponding Fouriertransformed data for standard In2O3 and C-In203-x, (f) XPS spectra of the In 3d core level peak regions of C-In203-x, inset: charge difference density of In2O3 with one $\mathrm{C}$ atom interstitial with one $\mathrm{O}$ atom vacancy. 
a

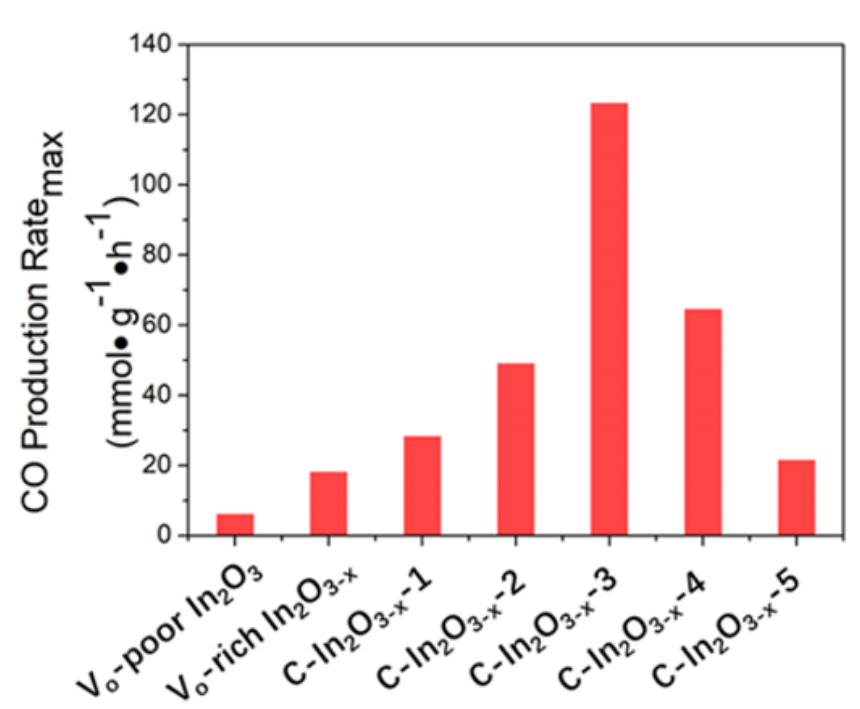

b

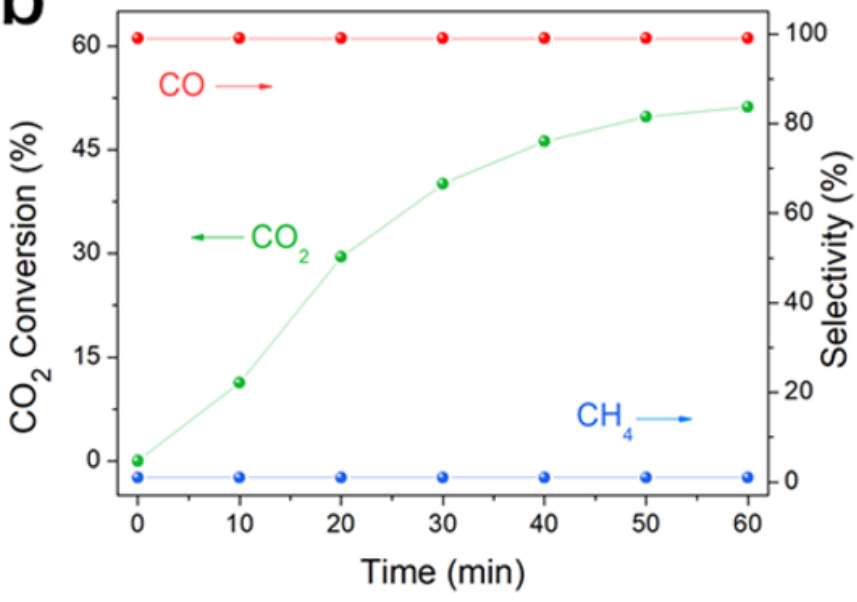

C

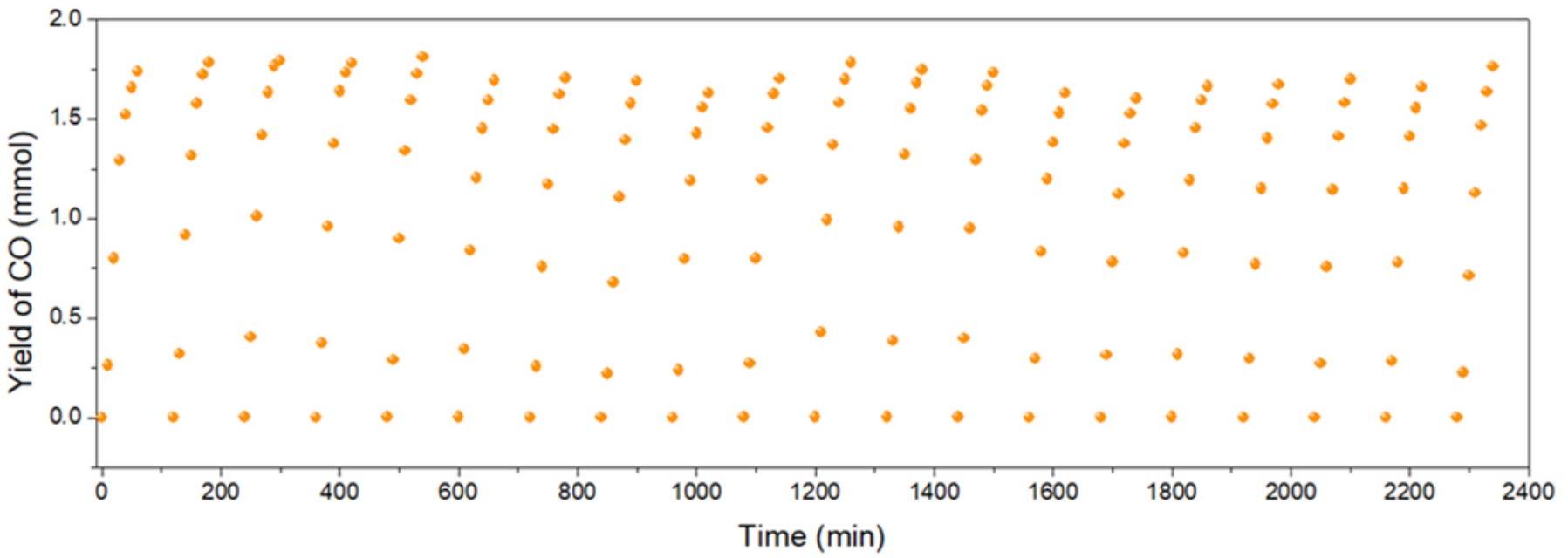

Figure 2

(a) CO production rates of Vo-poor In203, Vo-rich In203-x and C-In203-x with different carbon content, (b) photothermal CO2 conversion test and selectivity of C-In2O3-x-3. Reaction condition: 50 mg catalysts, H2: CO2=1:1, 300 W Xenon lamp, (c) Cyclic stability testing of C-In203-x-3. 
a

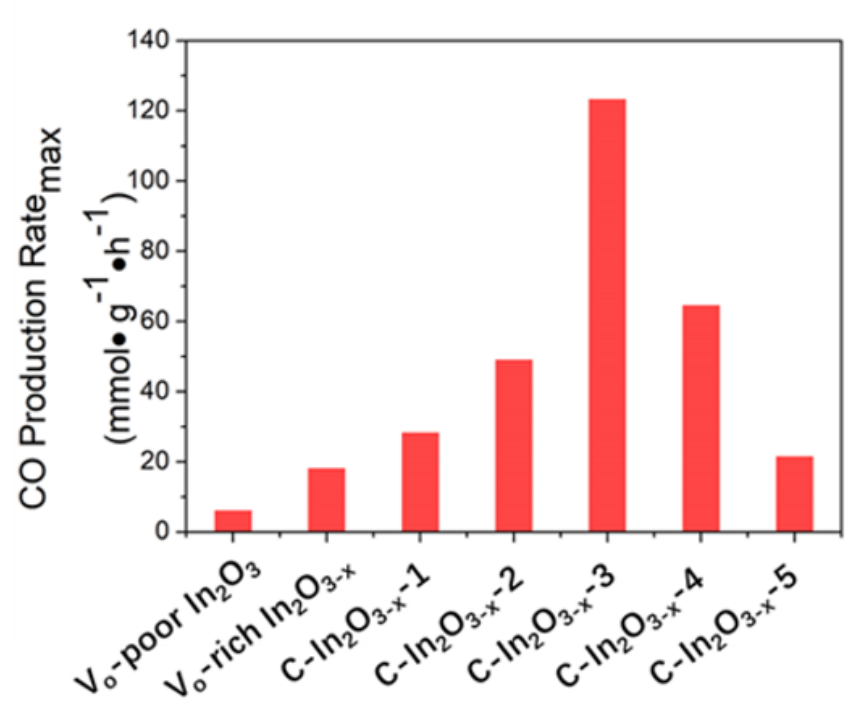

b

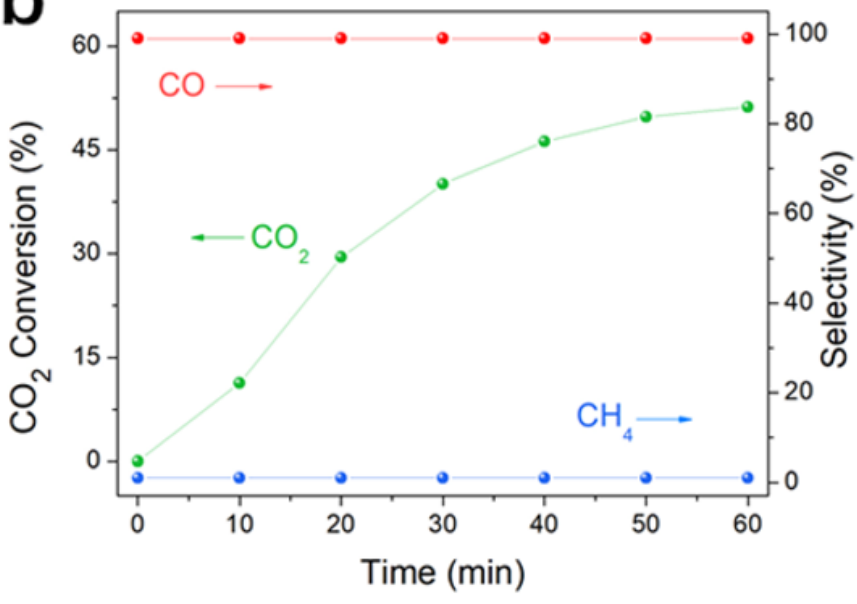

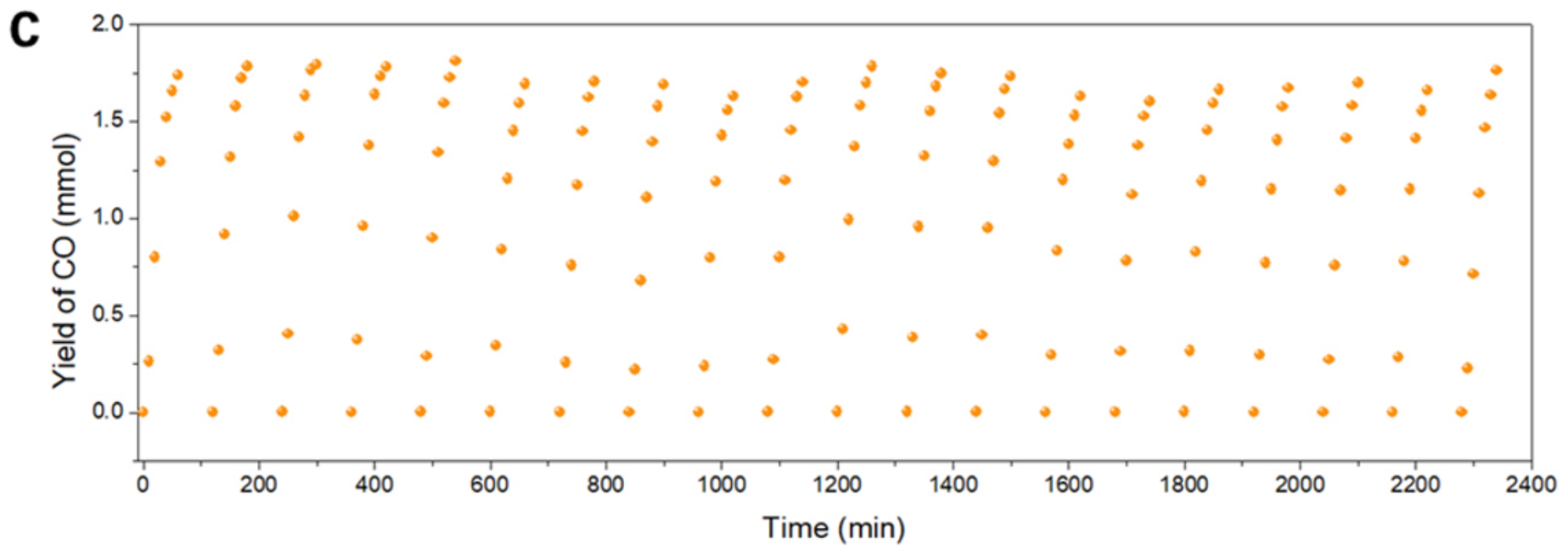

Figure 2

(a) CO production rates of Vo-poor In203, Vo-rich In203-x and C-In203-x with different carbon content, (b) photothermal CO2 conversion test and selectivity of C-In2O3-x-3. Reaction condition: 50 mg catalysts, H2: CO2=1:1, 300 W Xenon lamp, (c) Cyclic stability testing of C-In203-x-3. 

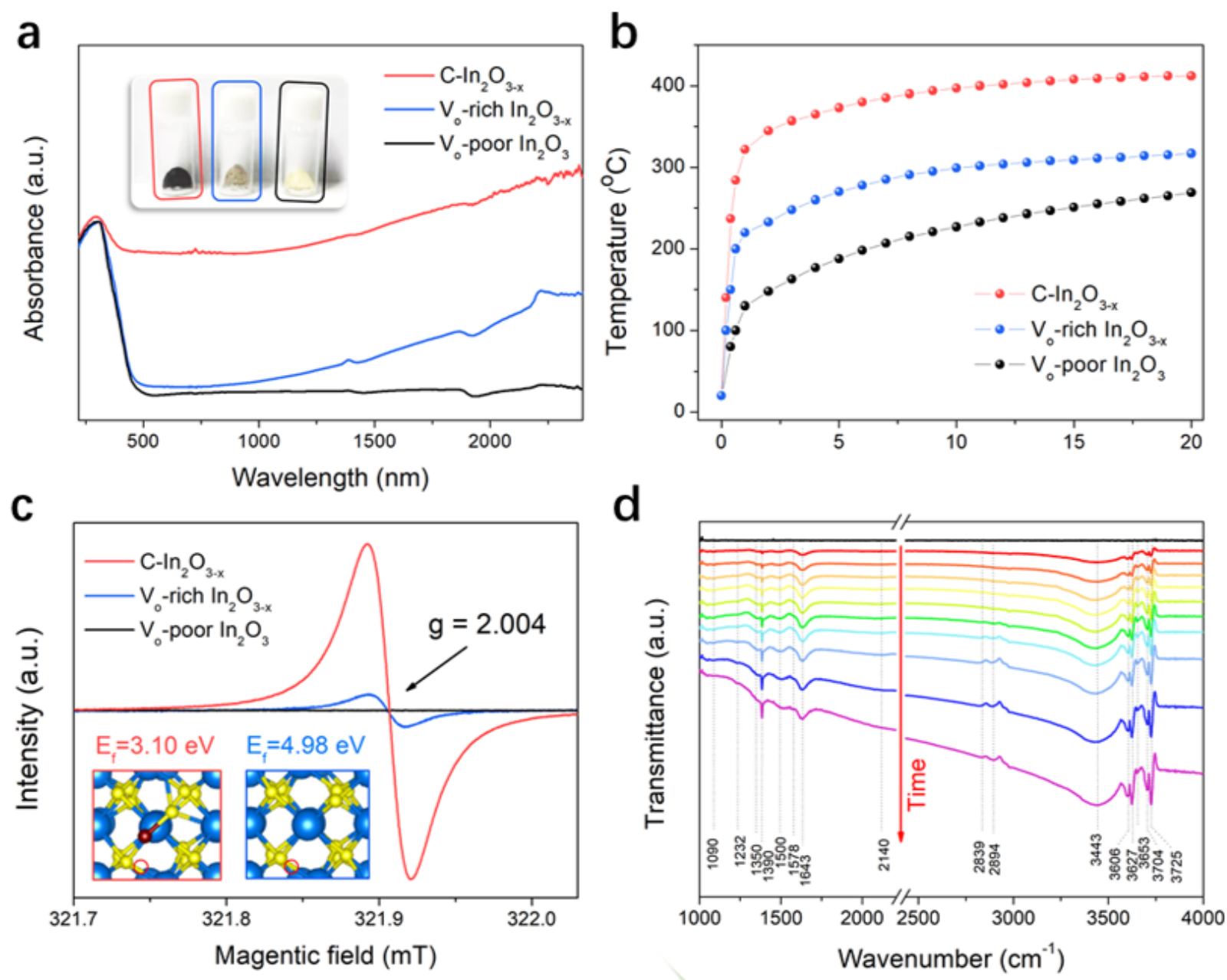

e

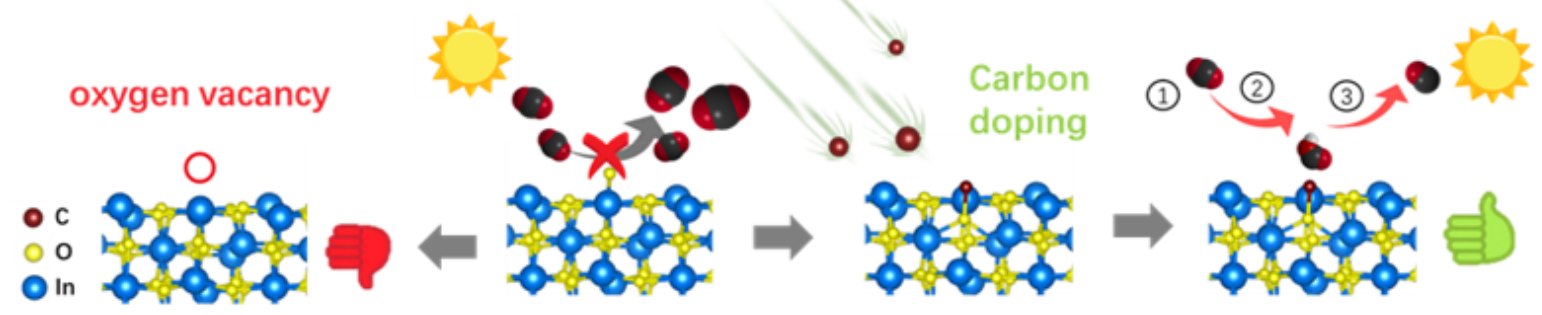

Figure 3

(a) UV- visible -NIR spectra, Inset: digital photo, (b) Temperature monitoring of photothermal conversion of CO2 system, (c) Electron spin resonance spectra, Inset: Oxygen vacancy formation energy of perfect In203 and Ci-In203, (d) in situ FT-IR spectra, (e) Advantages for $\mathrm{CO} 2$ photothermal reduction into CO over the C-In2O3-x. 

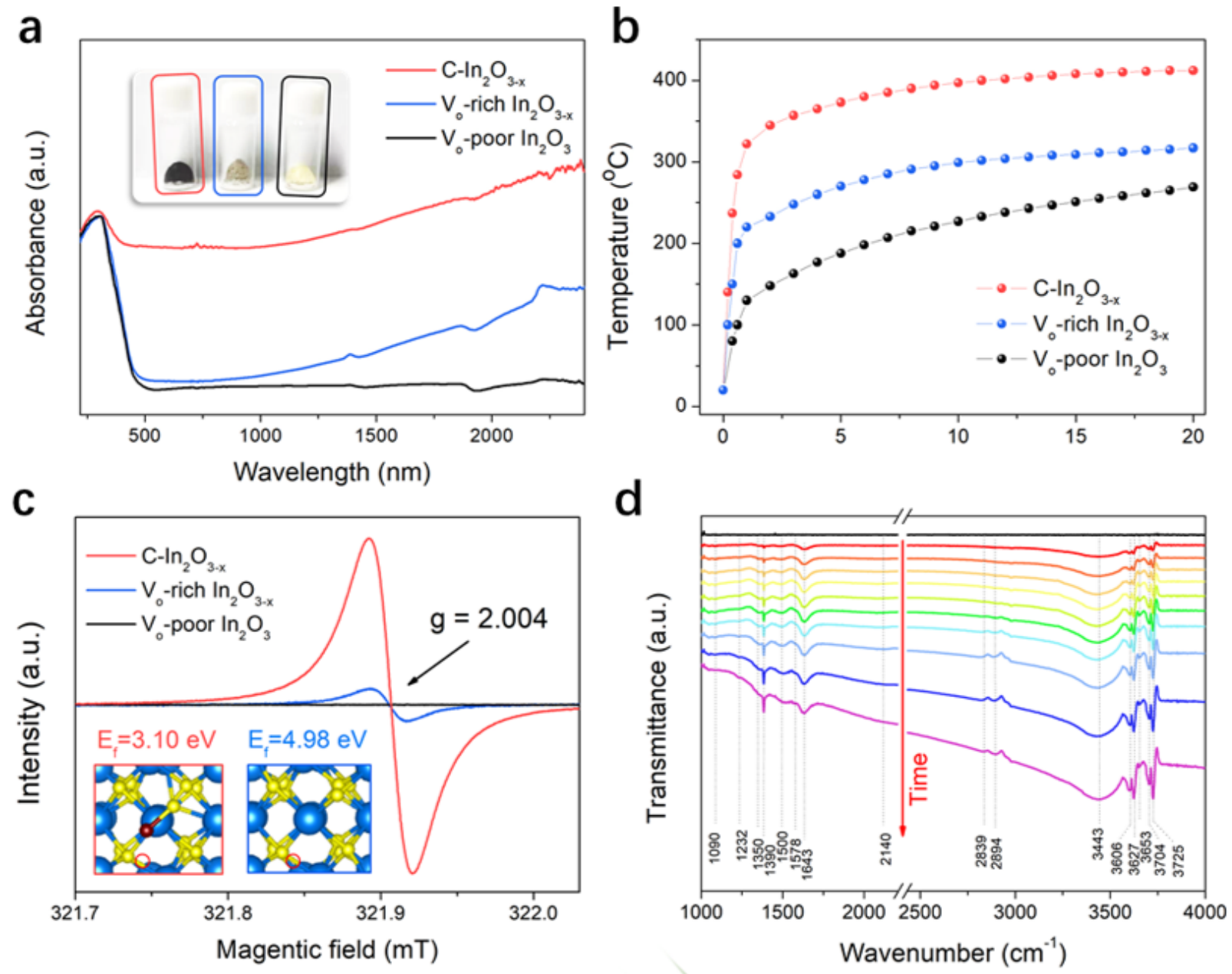

e

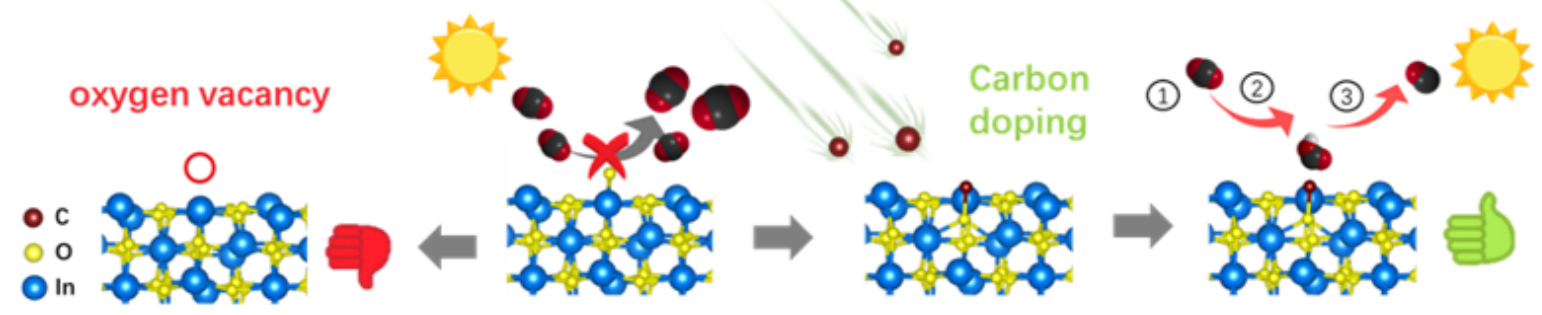

Figure 3

(a) UV- visible -NIR spectra, Inset: digital photo, (b) Temperature monitoring of photothermal conversion of CO2 system, (c) Electron spin resonance spectra, Inset: Oxygen vacancy formation energy of perfect In203 and Ci-In203, (d) in situ FT-IR spectra, (e) Advantages for CO2 photothermal reduction into CO over the C-In2O3-x.

\section{Supplementary Files}

This is a list of supplementary files associated with this preprint. Click to download.

- GUnpublishedWorksQiYHXXXXXXXXXNatureCommunicationsFinalVersionbeforeSubmissionSupplementarylnformation.docx

- GUnpublishedWorksQiYHXXXXXXXXXNatureCommunicationsFinalVersionbeforeSubmissionSupplementaryInformation.docx

- Scheme1.png

- Scheme1.png 